\title{
Manipulability Analysis of Human Arm Movements during the Operation of a Variable-Impedance Controlled Robot
}

\author{
Yoshiyuki TANAKA $^{1)}$ Naoki YAMADA ${ }^{2)} \quad$ Kazuo NISHIKAWA $^{2)}$ \\ Ichirou MASAMORI ${ }^{2)}$ Toshio TSUJI ${ }^{1)}$ \\ 1) Graduate School of Eng., Hiroshima Univ., Higashi-hiroshima, JAPAN, \{ytanaka, tsuji\}@bsys.hiroshima-u.ac.jp \\ 2) Mazda Motor Corporation, Hiroshima, JAPAN
}

\begin{abstract}
This paper focuses on a shift operation system of an automobile as an example of human-machine systems, and develops a virtual shift system using a variable-impedance controlled robot that can provide various types of shift dynamics. A series of operational experiments is carried out with different viscoelastic patterns installed in the virtual shift system by changing the relative position between the shift lever and the operator. Then, the relationship between operational feeling and human arm movements is investigated by using the force manipulability considering human joint-torque characteristics.

Index Terms - Human-machine systems, operational feeling, force manipulability, variable-impedance control
\end{abstract}

\section{INTRODUCTION}

A human can control dynamic properties of his/her own body naturally and effectively according to tasks by utilizing the perceived information of environmental characteristics. For example, in the door open-close task usually appearing in our daily activities, a human can carry out a smooth operation without feeling an excessive load by controlling his hand movements and arm configurations at the same time. In such a task, a human can realize efficient and appropriate motor control of his own body according to the cognitive environmental characteristics perceived through his sensory receptors. If the relationship between human sensation and dynamic properties of movements for environmental characteristics can be described quantitatively, it would be useful to design and develop a novel human-machine system in which the operator can manipulate the machine comfortably.

Research on the mechanisms of human arm movements, in which target tasks can be classified into free movements [1] and constrained movements with the interaction of a task environment [2]-[4], has been actively conducted so far. Most of these studies, however, deal with free movements, although human movements in daily activities are often constrained by task environments. There have been some reports on human arm movements during a crank rotation task restricted on the horizontal plane [2], [3], which is an example of the constrained tasks, but they do not take into account of the influence of environmental constraints, such as dynamic properties and a operational trajectory determined by the constrained mechanism, on human arm movements and operational feeling.

On the other hand, the development of an advanced robotic system has been anticipated for tasks such as tending patients and elderly persons in hospitals, assisting human workers in an office environment, and so on. In such human-robotic systems, a human operator often takes the initiative in executing tasks; in contrast, robots are required to assist the operator's movements. Therefore, such a system should be designed with careful consideration of the control properties and operational feeling of a human operator, as well as the control accuracy and performance of the system, to achieve natural cooperation between a human operator and a robot.

To cope with such prerequisite conditions, a method of constructing a human-machine system using an impedancecontrolled robot [5] has been discussed in the robotics field [6]-[11]. The basic concept is that the robot impedance properties are designed to ensure the stability of the whole system and the maneuverability for a human operator with consideration of human impedance. For example, Ikeura et al. [9] proposed a variable-impedance control method that switches the robot's impedance properties during a task. They also supposed that the maneuverability of a human-machine system could be improved by installing human impedance, which measured in the target task with two human operators, into the impedance-controlled robot. Moreover, Yamada et al. [10], [11] developed a skill assist system that varies robot impedance properties according to human movements to perform iterative tasks at a constant speed with high quality. However, these previous studies argue mainly on a design approach of robot impedance properties without consideration of the relationship between human force manipulability and operational feeling during tasks.

In this paper, a manual gear shift system of an automobile is focused on as an example of a human-machine system, and a virtual shift system using a variable-impedance controlled robot is developed that can provide various types of shift dynamics. Then, the relationship between operational feeling 
and human arm movements is investigated by using the force manipulability considering human joint-torque characteristics [12]. This paper is organized as follows: Section II explains the developed virtual shift system constructed with a variableimpedance controlled robot. In Section III, a series of operational experiments is carried out with different viscoelastic patterns installed in the virtual shift system by changing the relative position between the shift lever and the operator. Finally, Section IV investigates the relationship between the dynamic properties of human arm movements and the feel of operations by using a quantitative index.

\section{VIRTUAL SHIFT SYSTEM}

\section{A. Experimental apparatus}

Fig. 1 illustrates a schematic view of the virtual shift system developed in this paper. The system is composed of a direct-drive typed liner motor table with one degree of freedom (Nihon Thomson Corp., maximum force \pm 10 [kgf]) , a computer for controlling the motor table, a biofeedback display for providing the measured hand movements. The display is also utilized as a control interface for regulating dynamic properties of the virtual shift.

A shift knob and a six-axis force/torque sensor (BL. Autotech, Ltd., resolution ability: force $x$ axis, $y$ axis: 0.05 $[\mathrm{N}], z$ axis: $0.15[\mathrm{~N}]$, torque: $0.003[\mathrm{Nm}])$ are attached to the moving part of the linear motor table. The force sensor measures the hand force generated in shifting, and the hand position is measured by an encoder built in the table (encoder resolution: $2[\mu \mathrm{m}]$ ). Also, a stereo camera system (Quick MAG, Ouyou Keisoku Kenkyusyo INC.) is employed in order to observe human arm movements during shifting, which can detect the 3-D position of a color marker attached at a measurement point (maximum of 8 points) from two 2D image sequences taken by two CCD cameras in real time (sampling rate: $60[\mathrm{~Hz}]$ ).

\section{B. Control system}

Fig. 2 shows a block diagram of the virtual shift system, in which $x$ is the current position of the operator's hand (the shift knob) and $x_{d}$ is the target position, and $F$ is the hand force generated by the operator. In the robot impedance control part, tracking control is executed to make the shift knob follow a virtual target point $x_{v}$ calculated from the impedance filter with hand force $F$.

As a target operation in this paper, the shifting operation from Low gear to 2nd gear is discussed as an example of straight human movements with environmental constraints. To provide a realistic feeling of shifting, the liner motor table, in the virtual shift system, is controlled by the variableimpedance control with respect to the shift knob position $x$. So, its dynamic properties can be represented as follows:

$$
M \ddot{x}_{v}+B(x) \dot{x}_{v}+K(x)\left(x_{v}-x_{c}\right)=F,
$$

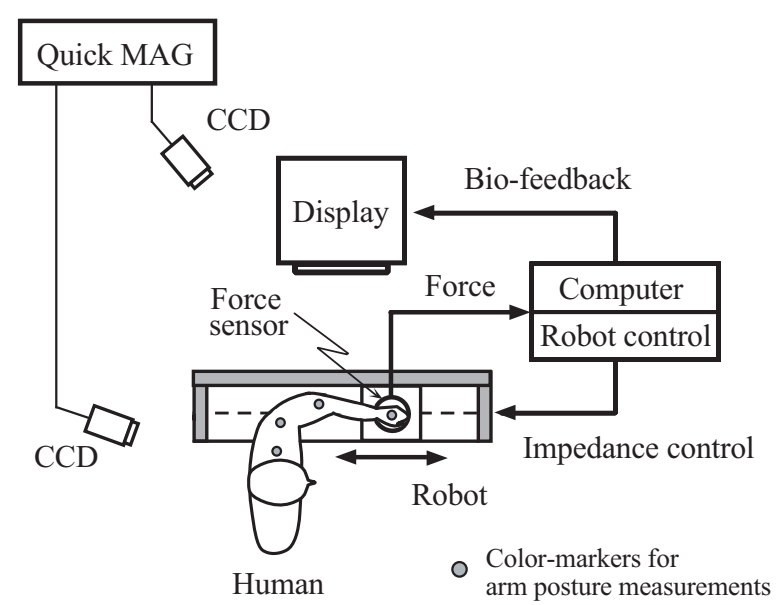

Fig. 1. Schematic illustration of a virtual shift system

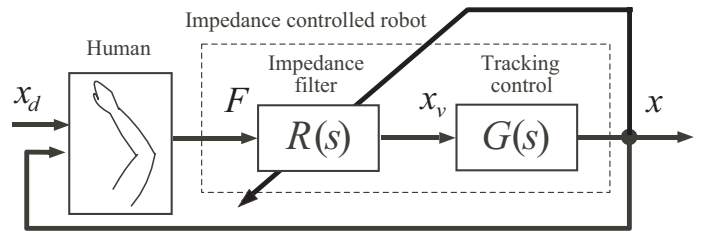

Fig. 2. Block diagram of the variable-impedance controlled robot

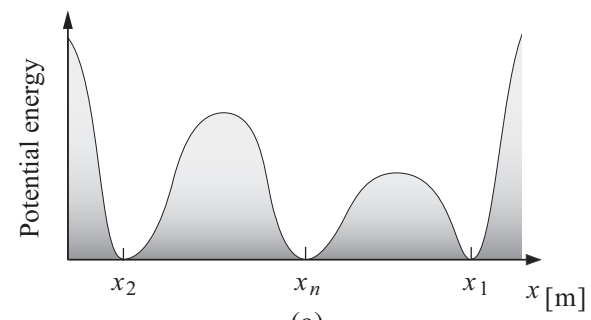

(a)

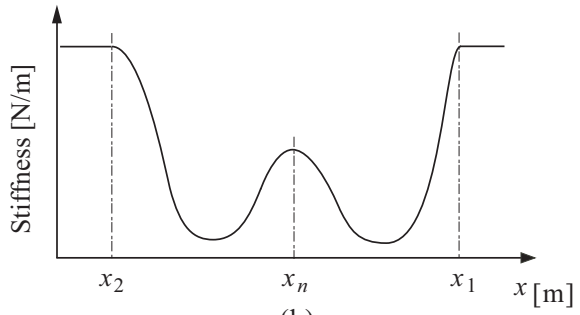

(b)

Fig. 3. Viscoelastic pattern of the virtual shift system

where $M$ is the inertia of a virtual shift; $B(x)$ and $K(x)$ the variable viscosity and stiffness as a function of the hand position $x$, respectively; and $x_{c}$ an equilibrium for $K(x)$.

Regarding the points of Low gear $x_{1}$, Neutral gear ( $\mathrm{N}$ gear, here after) $x_{n}$, and 2nd gear $x_{2}$ as the equilibrium $x_{c}$, in this virtual shift system, the profile of potential energy during the shifting operation from Low gear to 2nd gear can be drawn as Fig. 3(a). So, a viscoelastic pattern in each interval of 3 


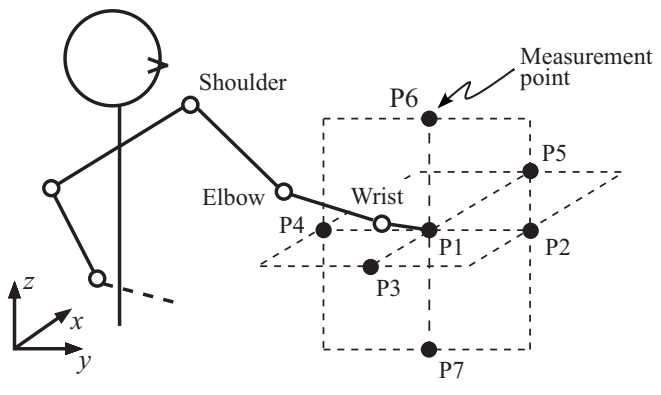

Fig. 4. Experimental condition on measurements

gears is designed with Gauss functions as shown in Fig. 3(b), while the equilibrium $x_{c}$ is switched by the following rule:

$$
x_{c}= \begin{cases}x_{1} & \left(x>x_{c 1}\right) \\ x_{n} & \left(x_{c 2} \leq x \leq x_{c 1}\right) \\ x_{2} & \left(x<x_{c 2}\right),\end{cases}
$$

where $x_{c 1}$ and $x_{c 2}$ represent the switching points of the equilibrium.

Installing an appropriate viscoelastic pattern with consideration of the engagement in shifting into the impedance control part, the virtual shift system can provide various kinds of dynamic properties of the shift lever to a human operator.

\section{EXPERIMENTS}

\section{A. Experimental method}

A human subject has a seat set parallel to the virtual shift system as shown in Fig. 1, and he is asked to move the shift knob from Low gear to 2nd gear after positioning $\mathrm{N}$ gear at the specified point. In this paper, we set seven points for each subject, $\mathrm{P} 1 \sim \mathrm{P} 7$, considering the individual differences of the subject's physical characteristics as shown in Fig. 4. The point where a subject could operate the virtual shift with a good feeling was determined as a base point P1 for each subject. A human upper arm is expressed by a serial link model with 7 rotational joints as shown in Fig. 5, and the subject's arm posture is measured during the shifting task.

A set of experiments was conducted with three righthanded subjects (male university students) under the measurement conditions as shown in Table I. In this paper, the distances between Point P1 and Points P2, P3 were set as $0.1[\mathrm{~m}]$ and $0.055[\mathrm{~m}]$, respectively, because of the system's structure, while the other points were set as 0.15 [m]. The virtual system was controlled with the sampling time $\Delta t=0.001[\mathrm{~s}]$, and the beginning and termination of hand movements was determined at the time when the norm of hand velocity were just over/under $1 \%$ of the maximum in each trial.

\section{B. Hand motion properties for viscoelastic patterns}

Three viscoelastic patterns employed in this paper are shown in the upper part of Fig. 6. Pattern 1 can provide

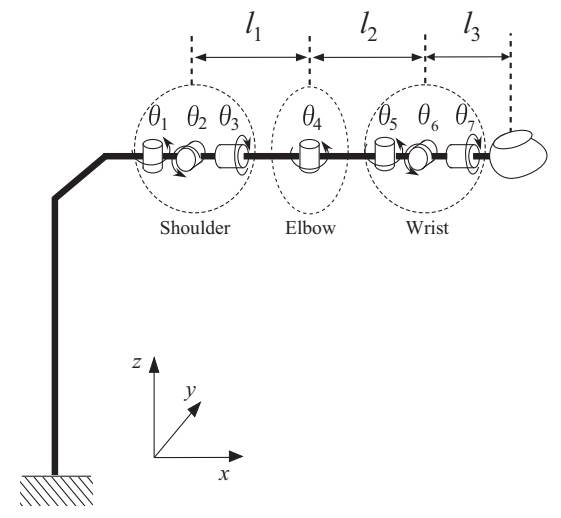

Fig. 5. Link model of a left upper extremity

TABLE I

BASE POINTS AND LINK LENGTHS OF THE UPPER ARM FOR EACH SUBJECT

\begin{tabular}{c|c|c|c|c|c|c}
\hline \hline \multirow{2}{*}{ Subject } & \multicolumn{3}{|c|}{$\begin{array}{c}\text { Link length of } \\
\text { the upper extremity }\end{array}$} & \multicolumn{3}{c}{$\begin{array}{c}\text { Relative position of P1 } \\
\text { from the shoulder joint }\end{array}$} \\
\cline { 2 - 7 } & $l_{1[\mathrm{~mm}]}$ & $l_{2[\mathrm{~mm}]}$ & $l_{3[\mathrm{~mm}]}$ & $x_{[\mathrm{mm}]}$ & $y_{[\mathrm{mm}]}$ & $z_{[\mathrm{mm}]}$ \\
\hline $\mathrm{A}$ & 280 & 235 & 80 & 270 & 390 & -155 \\
\hline $\mathrm{B}$ & 300 & 250 & 90 & 320 & 375 & -210 \\
\hline $\mathrm{C}$ & 280 & 250 & 80 & 335 & 350 & -230 \\
\hline \hline
\end{tabular}

a realistic shift feeling to a human operator, which was designed with a middle-aged male driver with excellent driving skills. The other two patterns were modified based on Pattern 1 by changing the maximum point of the viscosity profile. The feeling of drawing a shift knob to the shift gears is obtained by increasing the value of robot stiffness around the equilibriums, while the feeling of gear changes is by regulating the peak and magnitude of robot viscosity. The operational range is virtually produced by rising viscoelastic patterns as shown in Fig. 6. The distance between $\mathrm{N}$ gear and the others was set at $0.04[\mathrm{~m}]$, and the inertia of a shift lever $M$ was at $0.45[\mathrm{~kg}]$.

Force-to-stroke (F-S) profiles for each viscoelastic pattern measured during the shifting operation are presented in the bottom part of Fig. 6, where the horizontal axis denotes the hand position while the vertical axis denotes the operational force exerted by the subject's hand. All of the experimental results measured at the seven points, depicted in Fig. 4, are plotted in the figures. Measurement points did not have an influence on the F-S profile during the shifting operation, while viscoelastic patterns remarkably changed the characteristics of human hand movements. In addition, the peak of the F-S profiles appeared at the point where the robot viscosity takes the maximum value.

It can be found that the hand operational force has a peak and the change of F-S profiles is shaped around $\mathrm{N}$ gear under Pattern 1 providing realistic shift dynamics. On the other 

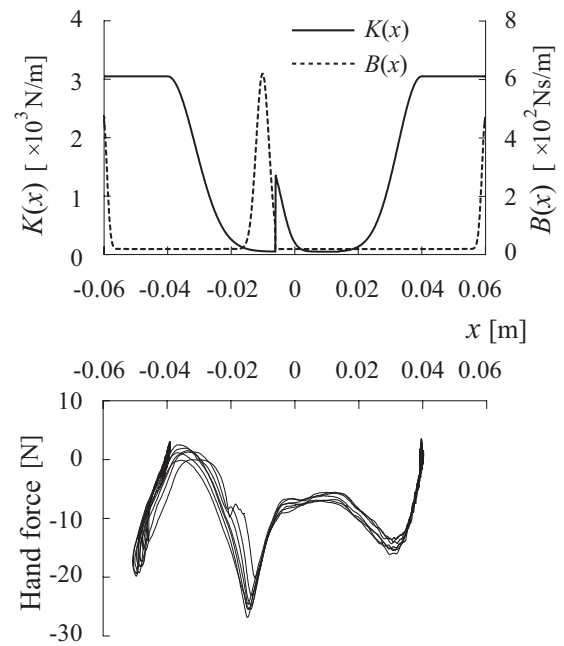

(a) Pattern1
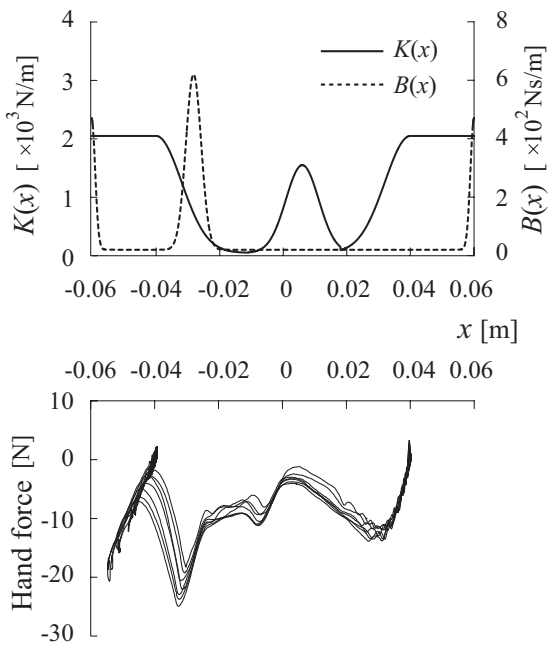

(b) Pattern 2

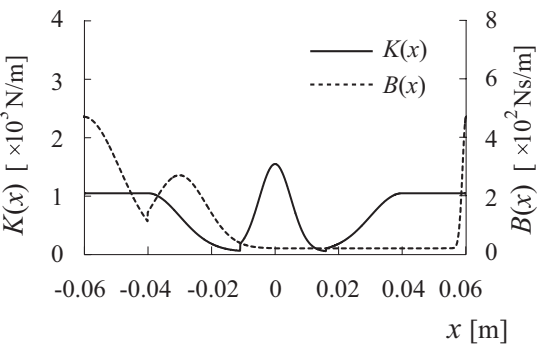

$\begin{array}{lllllll}-0.06 & -0.04 & -0.02 & 0 & 0.02 & 0.04 & 0.06\end{array}$

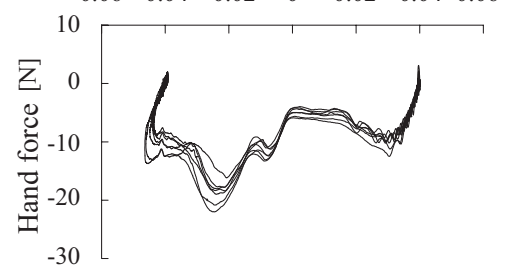

(c) Pattern 3

Fig. 6. Viscoelastic patterns installed in the virtual shift system and examples of the hand force profiles with respects to the hand positions by Subject A

hand, in the shifting operation with Pattern 2, double-peaked profiles appear between $\mathrm{N}$ gear and 2nd gear mainly because the peaks of robot stiffness and viscosity in Pattern 2 are set at a separated point. Thereby, an operator would find a twostep gear change during the shifting operation from Low gear to 2nd gear. Similar characteristics can be seen for Pattern 3, in which the F-S profiles become smooth since the viscosity pattern of Pattern 3 does not sharpen.

As shown in these experimental results, the developed virtual shift system can represent various types of shift dynamics, as well as realistic shift dynamics, by regulating the viscoelastic pattern installed into the variable-impedance controlled motor table. The following part of the present paper discusses the dynamic properties of human movements and the operational feeling during the shifting operation with Pattern 1.

\section{Shifting feels for operational positions}

Table II shows the mean and standard deviation (mean $\pm \mathrm{SD}$ ) of the evaluation results of operational feeling for all subjects. The number of trials in each measurement point was set at five, and the subjects were asked to evaluate operational feeling by five levels as; 5: "Very good", 4: "Good", 3: "Normal", 2: "Bad", 1: "Very bad." The operational feeling of the subjects changed according to the operational position for the same shift dynamics. It can be found that the evaluation of the operational feeling by all subjects becomes high for Point P1, while it provides poor evaluations for Points P5 and P7 in which the subjects had to stretch the upper arm.

Fig. 7 shows the changes of the arm postures observed during the shifting operation for Points P1, P4, and P5. Comparing among them, it can be found that the subject operates the virtual shift with tight arm postures for $\mathrm{P} 4$ since
TABLE II

OPERATIONAL FEELING FOR THE SHIFT POSITIONS

\begin{tabular}{c|c|c|c}
\hline \hline \multirow{2}{*}{$\begin{array}{c}\text { Shift } \\
\text { position }\end{array}$} & \multicolumn{3}{|c}{ Evaluation value } \\
\cline { 2 - 4 } & Subject A & Subject B & Subject C \\
\hline P1 & $4.8 \pm 0.45$ & $4.2 \pm 0.45$ & $4.0 \pm 1.22$ \\
\hline P2 & $3.8 \pm 0.84$ & $2.4 \pm 0.89$ & $2.8 \pm 1.10$ \\
\hline P3 & $4.6 \pm 0.55$ & $3.8 \pm 0.45$ & $3.6 \pm 0.55$ \\
\hline P4 & $3.2 \pm 1.30$ & $2.8 \pm 0.45$ & $2.6 \pm 0.55$ \\
\hline P5 & $3.2 \pm 0.45$ & $1.6 \pm 0.55$ & $1.2 \pm 0.45$ \\
\hline P6 & $3.6 \pm 0.89$ & $2.6 \pm 0.55$ & $2.2 \pm 0.45$ \\
\hline P7 & $3.2 \pm 0.84$ & $1.4 \pm 0.89$ & $1.0 \pm 0.00$ \\
\hline \hline
\end{tabular}

the elbow joint moves back to the shoulder joint, and that he executes the task at a position far from the shoulder point with a stretched arm posture for P5.

These experimental findings from the qualitative results suppose that the subject's feeling of the shifting operation is much affected by the arm posture according to the operational position. Next session analyzes the relationship between characteristics of human arm movements and operational feelings during the shifting operation by using a force manipulability ellipse considering human joint-torque properties [12].

\section{SHIFTING FEEL AND HAND FORCE MANIPULABILITY}

\section{A. Human force manipulability [12]}

The relationship between the force exerted on the tip $f \in$ $\Re^{m}$ and the joint-torque $\tau \in \Re^{n}$ under the link model of a human upper arm, as shown in Fig. 5, can be given by

$$
\boldsymbol{\tau}=\boldsymbol{J}(\boldsymbol{\theta})^{T} \boldsymbol{f}
$$




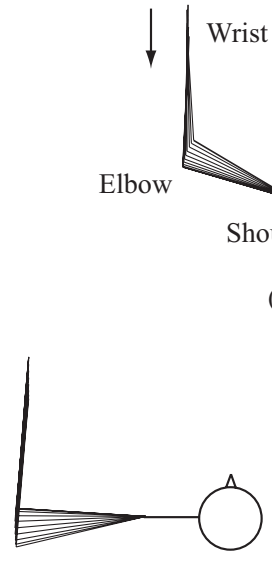

(b) P4

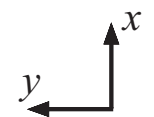

(a) P1

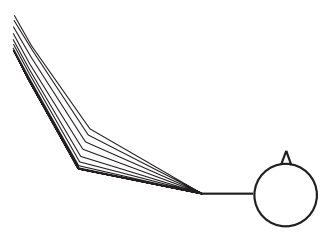

(c) P5
Fig. 7. Stick pictures of the upper extremity for Points P1, P4, and P5 during the shifting operation by Subject A

where $\boldsymbol{J}(\boldsymbol{\theta}) \in \Re^{m \times n}$ is the Jacobian matrix on the end-point position $\boldsymbol{x} \in \Re^{m}$ with respect to $\boldsymbol{\theta} \in \Re^{n}$.

Let us introduce the joint-torque activation level $\boldsymbol{\alpha}=$ $\left(\alpha_{1}, \alpha_{2}, \cdots, \alpha_{n}\right)^{T} \in \Re^{n}$, in which $\left|\alpha_{i}\right| \leq 1$ represents the ratio of $i$-th joint-torque to the maximum torque under the maximum voluntary contraction (MVC) and its sign denotes the joint rotational direction (the flexional direction is defined as positive). In this paper, the joint-torque vector $\tau$ is then expressed with $\boldsymbol{\alpha}$ because the muscle tension is proportional to its muscle activation level as follows:

$$
\tau=\boldsymbol{T}(\theta) \alpha
$$

where $\boldsymbol{T}(\boldsymbol{\theta})=\operatorname{diag} .\left(\tau_{1 j}^{\max }\left(\theta_{1}\right), \tau_{2 j}^{\max }\left(\theta_{2}\right), \cdots, \tau_{n j}^{\max }\left(\theta_{n}\right)\right)^{T}$ $\in \Re^{n \times n}(j \in\{f, e\}$; the suffix $f$ and $e$ indicate the flexional direction and the extensional direction, respectively). Each diagonal element of $\boldsymbol{T}(\boldsymbol{\theta})$ indicates an absolute value of the maximum joint-torque at an angle $\theta_{i}$.

From (3) and (4), a set of hand forces $f$ generating by the muscles within $\|\boldsymbol{\alpha}\| \leq 1$ makes an $m$ dimensional ellipsoid represented by

$$
\boldsymbol{f}^{T}\left(\boldsymbol{J} \boldsymbol{T}^{-1}\right)\left(\boldsymbol{J} \boldsymbol{T}^{-1}\right)^{T} \boldsymbol{f} \leq 1
$$

This human force manipulability ellipsoid (HFME) given in (5) corresponds to the transformed FME by the matrix $\boldsymbol{T}(\boldsymbol{\theta})$ that reflects the characteristics of human joint-torque. The size and shape of HFME can be utilized as a performance index in generating hand force according to the operational direction under the measured arm posture $\boldsymbol{\theta}$. For example, large operational force can be easily exerted in the major axis direction, while it is difficult toward the minor axis direction.

\section{B. Force manipulability during the shifting operation}

Fig. 8 shows examples of the experimental results for the measurement points P1, P4, and P5, where hand force

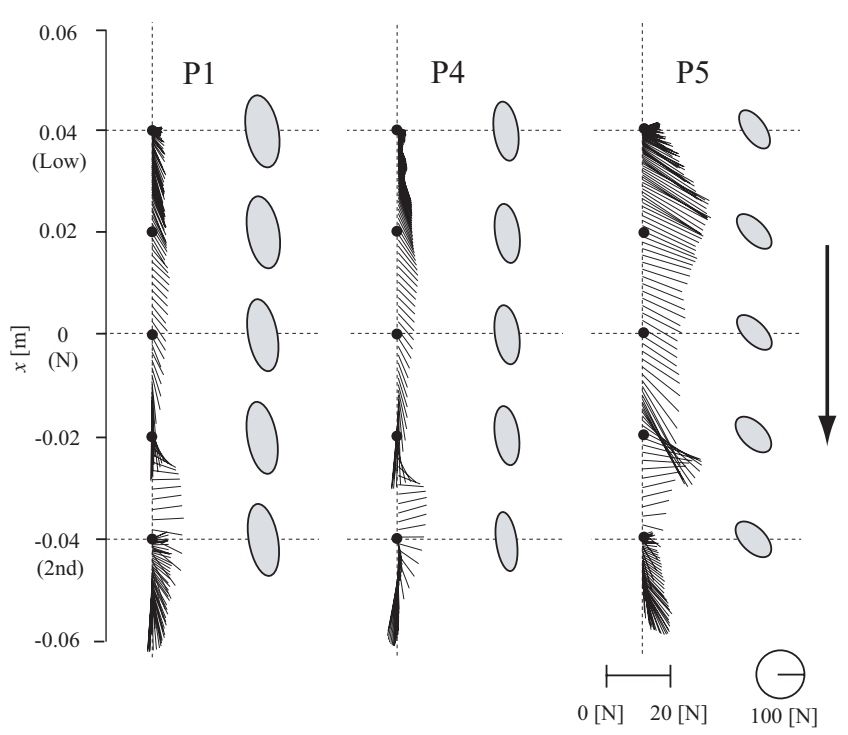

Fig. 8. Characteristics of hand movements during the shifting operation for Points P1, P4, and P5 under Pattern 2 by Subject A

vectors and HFMEs on the operational plane are presented. Five HFMEs were calculated from the subject's arm postures observed at the three gear positions and the two middle points between gears $(x=0.04,0.02,0,-0.02,-0.04[\mathrm{~m}])$ during the shifting operation.

Directing our attention to the hand force vectors during the shifting operation, it can be found that the subject shifts the knob with small hand forces for P1 where he did the shifting operation comfortably. While he always generated large hand forces towards the different directions from the operational direction for P5, in which he felt an uncomfortable operational feeling. This fact supposes that the subject's operational feeling became awkward because of the large hand force including elements that do not contribute to the shifting operation directly. Although the subject reported different operational feelings for Points P1 and P4, there does not exist, however, much differences on the hand force vectors, such as the norm and the direction, measured for each measurement point during the operaiton.

On the other hand, focusing on the characteristics of HFMEs, we can find that the size of HFME for Point P1 is obviously larger than one for Points P4 and P5, and that the relative angle between the major axis of HFME and the operational direction is small for Points $\mathrm{P} 1$ and $\mathrm{P} 4$ while it becomes large for P5. In addition, comparing the HFMEs calculated for Points P1 and P4, the HFME for P4 is smaller on the whole. Thereby, it was relatively difficult for a human subject to generate the large hand force required for the task under the arm configurations for $\mathrm{P} 4$, and so his operational feeling became awkward for this operational condition.

Based on the above analysis and the consideration, it can be ascertained that there exists a close relationship 
between the operational feeling of a human operator and the characteristics of HFME calculated with the observed arm posture during the operation. Finally, the operational feeling is examined from the arm posture by using the quantitative index $E$ defined with HFME by

$$
E=S \cos (|\psi|)
$$

where $S$ denotes the size of HFME; and $\psi\left(-\frac{\pi}{2} \leq \psi \leq\right.$ $\left.\frac{\pi}{2}\right)$ the relative angle of the major axis of HFMEs for the operational direction. The operational feeling would become better as the value of $E$ increased.

Fig. 9 shows the evaluation results with the quantitative index $E$ by using the mean values of $S$ and $\psi$ at the five points as depicted in Fig. 8 for all subjects, where the horizontal axis arranges the measurement points according to the subjective evaluation results for each subject reported in Table. II. The value of $E$ becomes maximum for P1 where all subjects reported that they felt the best operational feelings within the specified experimental conditions, while it becomes lower for P5 and P7. This fact supposes that the subjects could find out the point where the index $E$ would become large intuitively. Consequently, it may be possible to predict and evaluate the operational feeling of a human subject during the shifting operation only from the arm postures by using the index $E$ based on HFME.

\section{CONCLUSion}

This paper has developed a virtual shift system using a variable-impedance control method, as an example of a human-machine system to investigate human hand movements and operational feeling according to operational conditions. Then, we analyzed the relationship between human force manipulability and operational feeling quantitatively by using HFME, and found that it may be possible to estimate human operational feeling through the characteristics of human force manipulability ellipses calculated from the arm posture in the shifting motion.

Future research will be directed to examine human hand movements and operational feelings for curved trajectories, as well as straight ones, during the shifting operation not only on the horizontal operational plane but also on the slope plane. We also plan to investigate human impedance properties during a control task of a human-machine system [13], including the virtual shift system, in order to develop a novel design method of such robotic systems considering human compliant movements.

The authors would like to thank Mr. M.Sasaki at Hiroshima University, Dr. T.Nouzawa and Mr. K.Miyamoto at Mazda Motor Corporation. This research work was supported in part by a Grant-in-Aid for Scientific Research from the Japanese Ministry of Education, Science and Culture (15360226 and 16760203).

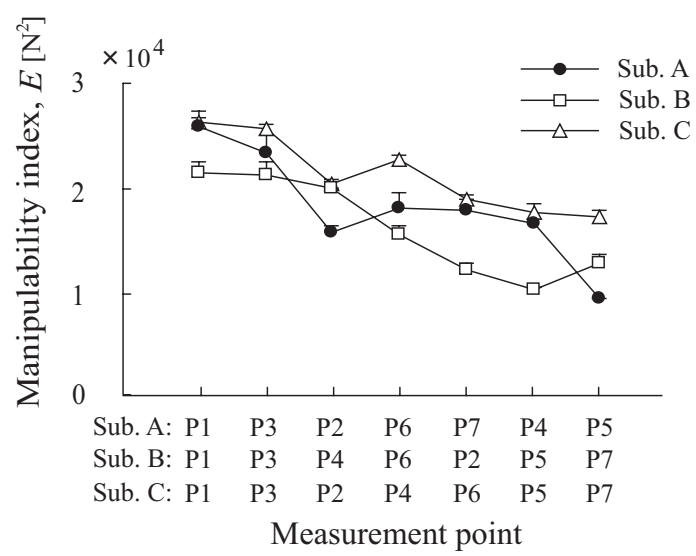

Fig. 9. Changes of the index value $E$ according to the measurement points for all subjects

\section{REFERENCES}

[1] P. G. Morasso: "Spatial Control of Arm Movements," Experimental Brain Rsearch, Vol. 42, pp. 223-227, 1981.

[2] K. Ito, T. Tsuji and M. Sugino: "Impedance Regulation in Human Movements During a Rotation Task," Journal of Robotics and Mechatronics, Vol. 3, No. 6, pp. 455-462, 1991.

[3] K. Ohta, M. M. Svinin, Z. W. Luo, S. Hosoe, and R. Laboissière: "Optimal trajectory formation of constrained human arm reaching movements," Biological Cybernetics, Vol. 91, pp. 23-36, 2004.

[4] T. Tsuji, Y. Tanaka, M. Kaneko: "Biomimetic trajectory generation based on human movements with a nonholonomic constraint," IEEE Transactions on Systems, Man, and Cybernetics-Part A: Systems and Humans, Vol.32, No.6, pp.773-779, November 2002.

[5] N. Hogan: "Impedance control: An approach to manipulation," Parts I, II, III, ASME Journal of Dynamic Systems, Measurement, and Control, Vol.107, No.1, pp. 1-24, 1985.

[6] H. Kazerooni: "Human-robot interaction via the transfer of power and information signals," IEEE Transactions on Systems, Man, and Cybernetics, Vol.20, No.2, pp. 450-463, March/April 1990.

[7] K. Kosuge, Y. Fujisawa, and T. Fukuda: "Control of mechanical system with man-machine interaction," in Proceedings of IEEE International Conference on Intelligent Robots and Systems, pp. 87-92, 1992.

[8] T. Tsuji, Y. Tanaka, and M. Kaneko: "Tracking control properties of human-robot systems," in Proceedings of the 1st International Conference on Information Technology in Mechatronics, pp. 77-83, 2001.

[9] R. Ikeura and H. Inooka: "Variable impedance control of a robot for cooperation with a human," Proceedings of the 1995 IEEE International Conference on Robotics and Automation, pp. 3097-3102, 1995.

[10] Y. Yamada, H. Konosu, T. Morizano, and Y. Umetani: "Proposal of skill-assist: A system of assisting human workers by reflecting their skills in positioning tasks," Proceedings of the 1999 IEEE International Conference on Systems, Man, and Cybernetics, IV11-16, 1999.

[11] Y. Yamada, H. Daitoh, T. Sakai, and Y. Umetani: "Proposal of a human interface technique for reflecting the operator's intentionality in human/robot collaborative conveyance tasks," Transactions of the Japan Society of Mechanical Engineers, Vol. 67, No.665-C, pp. 10691076, 2001.

[12] Y. Tanaka, N. Yamada, I. Msamori, and T. Tsuji: "Manipulability Analysis of Lower Extremities Based on Human Joint-Torque Characteristics," in Proceedings of the 2nd International Symposium on Measurement, Analysis and Modeling of Human Functions, pp. 261266, 2004.

[13] T. Tsuji, Y. Takeda, and Y. Tanaka: "Analysis of mechanical impedance in human arm movements using a virtual tennis system," Biological Cybernetics, Vol.91, No.5, pp.295-305, 2004. 